Boletín de la Sociedad Geológica Mexicana

Volumen 61, NÚM. 3, 2009, P. 485-490

NOTA BREVE

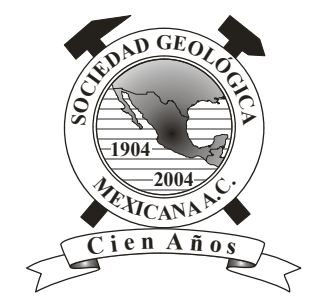

\title{
Contribuciones Geológicas de Charles Darwin en El origen de las especies
}

\author{
José Lugo Hubp
}

${ }^{1}$ Instituto de Geografía, UNAM. Ciudad Universitaria.04510 México, D.F.

*lugoh@servidor.unam.mx

\section{Resumen}

La obra cumbre de Charles Darwin (1809-1882), El Origen de las Especies (1859), a 150 años de su aparición sigue siendo uno de los libros más vendidos de la historia. Cuatro de los 15 capítulos son de orientación geológica, donde se presenta el análisis de los procesos de la sedimentación en el océano, su duración en el tiempo que determina el espesor de las unidades sedimentarias, los movimientos de levantamiento y hundimiento, la profundidad a la que ocurren y todo relacionado con la presencia o ausencia de fósiles, la erosión posterior en tierra firme y el tiempo necesario. Esto llevó a Darwin, al igual que a Charles Lyell, a suponer una edad de la Tierra de por lo menos 300 millones de años, algo inaceptable en aquella época. Darwin explicó el poblamiento del planeta por migraciones, donde las diversas condiciones geográficas y el tiempo, influyen en la transformación de los organismos. Acabó con el concepto del creacionismo que se debe a una concepción de la edad de la Tierra de algunos miles de años, donde la evolución no tiene cabida. Darwin hizo contribuciones importantes en la geología, como el origen de los atolones, los movimientos tectónicos presentes en la Cordillera de los Andes y otras. La geología posterior a Darwin ha reafirmado y fortalecido la teoría de la evolución.

Palabras clave: Charles Darwin, Geología, Paleontología, Paleobiogeografía, edad de la Tierra

\section{Abstract}

One hundred and fifty years after its appearance, Charles Darwin's (1809-1882) master work, On the Origin of Species (1859), continues to be one of the best selling books of all time. Four of the 15 chapters focus on geology, presenting analyses of oceanic sedimentation processes, which deal with the relationship between time and the thickness of sedimentary units, uplifting and sinking movements, the depth at which these processes take place, and all aspects related to the presence or absence of fossils, subsequent erosion on dry land, and the length of time necessary for these processes to take place. This work led Darwin, like Charles Lyell before him, to suppose that the Earth must be at least 300 million years old, something which was inconceivable at the time. Darwin explained the habitation of the planet through migrations, whereby diverse geographical conditions, combined with time, influenced the changes the organisms have undergone. He put to an end the idea of creationism, a concept in which the Earth is conceived as being a few thousand years old, and one which therefore leaves no place for evolution. Darwin made important contributions to geology in such areas as the origin of atolls, and tectonic movements in the Andes, among others. Subsequent geological research has reaffirmed and strengthened the theory of evolution.

Key words: Charles Darwin, Geology, Paleontology, Paleobiogeography, Earth's age 


\section{Introducción}

En el año 2009 se celebraron dos aniversarios relacionados con Charles Darwin, el 12 de febrero se cumplieron 200 años de su nacimiento y el 24 de noviembre 150 años de la publicación de El origen de las especies, cuyo título original en inglés fue $O n$ the origin of species by means of natural selection or the preservation of favoured races in the struggle for life. Darwin hizo una de las contribuciones al conocimiento más importantes en la historia. Con mucha razón la teoría de la selección natural, que reforzó a la teoría de la evolución, es comparada con lo que en su momento aportaron Nicolás Copérnico, Galileo Galilei e Isaac Newton.

Los conceptos sobre la evolución de los organismos tienen su origen, por lo menos en la cultura griega, algunos siglos antes de nuestra era. Posteriormente fue fundamentada en el siglo XVIII y principios del XIX por los naturalistas Georges Louis Lecrec, conde de Buffon (1707-1788), Erasmus Darwin (1731-1802) -abuelo de Charles- y Jean Baptiste Lamarck (1744-1829). Este último es considerado el padre de la teoría de la evolución, pero la explicación que dio sobre cómo se producen los cambios en los organismos tuvo poca aceptación en el medio científico.

Mucho se ha escrito sobre Darwin. Pocas obras científicas han sido tan leídas, lo que continúa hoy día, como el libro clásico acerca de la evolución. Las ediciones se repiten en el mundo en cerca de 30 idiomas. Además del interés del tema, favorece su divulgación el hecho de que es un libro accesible a todo público.

El origen de las especies incluye una reseña histórica, una introducción y 15 capítulos, el último trata de las conclusiones. La parte geológica se concentra en los capítulos X a XIII (De la imperfección del registro geológico, De la sucesión geológica de los seres orgánicos y De la distribución geográfica-XII y XIII-).

Este artículo es resultado de la lectura de El origen de las especies, del que se extrae el contenido geológico principal expuesto por su autor (con letra más pequeña se inserta parte del texto correspondiente). Es común que se hable y escriba sobre Darwin el biólogo y pocas veces se le menciona como un geólogo que hizo aportaciones valiosas al conocimiento de la Tierra. Los conceptos geológicos de Darwin están contenidos en varias de sus publicaciones, resultado de su viaje en el Beagle. En El origen de las especies la geología está enfocada a fundamentar la teoría de la selección natural. Las fuentes bibliográficas son el libro mencionado de la Editorial Porrúa (2004), con una amplia introducción de R.E. Leakey (p. VII-LV). Como complemento se consultaron otras publicaciones, originales en español o traducidas del inglés. La vida de Charles Darwin es un tema tratado entre otras obras recientes, por Gribbin (2003), Sarukhán (1988) y Torres (1995); Romer (1985) analiza la interpretación que da a los fósiles; la teoría sobre la formación de los atolones es expuesta por Yonge (1985), también por Sarukhán; Farrington como historiador de la ciencia hace elogios y críticas; algunos estudios temáticos son presentados en obras por Ruiz (1998 y 2002), la primera relacionada con filosofía de la ciencia, la segunda es un enfoque histórico que incluye los conceptos de científicos mexicanos en el pasado sobre la teoría de la selección natural. Una obra reciente que estudia a Darwin como geólogo se debe a Herbert (2005).

Darwin a diferencia de los hombres de ciencia que lo precedieron, vivió una época de desarrollo del conocimiento mucho más rica, en el entorno y tiempos favorables para afrontar el por qué de la biodiversidad. Y precisamente, de manera independiente llegó a las mismas conclusiones que el naturalista británico Alfred Wallace (1823-1913), quien caballeroso cedió el mérito a Charles Darwin.

Mi trabajo está ahora casi terminado; pero como todavía me llevará varios años completarlo y como mi salud está lejos de ser fuerte, se me ha instado a publicar este fragmento. Me he sentido más especialmente inducido a ello porque Mr. Wallace, que ahora está estudiando la historia natural del archipiélago malayo, ha llegado casi exactamente a las mismas conclusiones generales que yo sobre el origen de las especies (p. 3).

La teoría de la selección natural pudo estar en la imprenta por lo menos 15 años antes de la fecha en que se publicó y si no pasó de 1859 fue gracias a Wallace. El hecho es que tarde o temprano algún estudioso de la naturaleza hubiera llegado a lo mismo, y posiblemente antes de terminar el siglo XIX. Este concepto, como muchos otros (el origen de los sismos, la formación de las montañas o las glaciaciones) se llegó a explicar de manera racional por la observación directa en la naturaleza, ahí estaba todo escrito, sólo era necesario descifrar lo que decían el relieve terrestre, minerales, rocas, fósiles, plantas, animales y el hombre.

Darwin tuvo la oportunidad, siendo joven, con casi 22 años, de viajar de fines de 1831 a octubre de 1836 en el barco Beagle, acompañando al capitán y astrónomo Robert Fitzroy. Los lugares que visitaron, hoy día sabemos que son de un gran interés geológico y biológico, contrastantes entre sí: las islas Galápagos, los Andes, los arrecifes australianos. Todo esto permitió al investigador hacer una cantidad asombrosa de observaciones en El origen de las especies.

La obra de Darwin es esencialmente biológica, pero no era posible entender el problema de la evolución sin el complemento de la geología. Este es un punto importante, el hecho que Darwin estuvo en el lugar correcto en el momento preciso. De manera simultánea nació la geología moderna con Charles Lyell (1797-1875), aunque tiene un antecedente importante en la obra de James Hutton (1726-1797). Lyell inició la publicación de un tratado de geología, el primer volumen en 1830, mismo que fue fundamental para Darwin, lo llevó consigo en el Beagle y en 1832 recibió el segundo volumen en Sudamérica. Con el tiempo ambos naturalistas llevaron una estrecha amistad basada en el interés de ambos por la naturaleza. Lyell contribuyó a enriquecer los conocimientos geológicos de Darwin, pero también a la inversa, éste explicó el origen de los arrecifes coralinos, los atolones, en una publicación de 1842, tema que era un dolor de cabeza para los geólogos 
de la época, además de cambiar las ideas creacionistas de Lyell de la vida en la Tierra por las de la evolución, lo que ocurrió sólo en la década de 1860.

Además de la parte biológica referida a flora y fauna, estudiada en la naturaleza y en las obras científicas, ya entonces sustanciosas, Darwin se apoyó en un estudio de Thomas Robert Malthus sobre la herencia:

Es la doctrina de Malthus aplicada a todo el reino animal y vegetal. Cómo nacen de cada especie muchos más individuos de los que pueden sobrevivir; y cómo, en consecuencia, se repite a menudo la lucha por la existencia (p. 5).

Los temas relacionados con la geología contenidos en el libro en cuestión, se resumen en cuatro subtítulos: La edad de la Tierra, Los fósiles, Evolución geológica y Distribución geográfica.

\section{La edad de la Tierra}

Fue el obstáculo principal para entender nuestro planeta y la vida que sostiene. Las disciplinas correspondientes, Geología y Biología, sólo pudieron madurar a lo largo del siglo XIX, cuando se fue aclarando que el tiempo correspondiente debía ser de cientos de millones de años. La explicación de la vida por uno o más actos de creación respondía a un concepto de un tiempo breve, de pocos miles de años. La teoría de la evolución no encajaba, en cambio, era algo lógico y racional asociarla al tiempo de millones de años. Darwin encontró en la geología la prueba del tiempo de cientos de millones de años, lo que reconoció en las rocas, más que en los organismos, y naturalmente, en las publicaciones de otros científicos:

La extensión y espesor de nuestras formaciones sedimentarias son el resultado y la medida de la erosión que la corteza de la Tierra ha sufrido en todas partes. Por consiguiente, un hombre debería examinar por sí mismo las grandes pilas de estratos superpuestos y observar a los riachos que arrastran barro y a las olas gastando los riscos marinos para comprender algo sobre la duración del tiempo pasado, cuyos monumentos vemos alrededor de nosotros (p. 269).

En la segunda mitad del siglo XIX el físico británico William Thomson (1824-1907), mejor conocido como Lord Kelvin, había calculado la edad de la Tierra tomando en cuenta la temperatura conocida y el tiempo que debió transcurrir desde que estuvo en estado incandescente:

Sir W. Thompson llega a la conclusión de que la consolidación de la corteza difícilmente puede haberse producido hace menos de veinte millones de años, o más de cuatrocientos millones; y que probablemente ha ocurrido hace por lo menos noventa y ocho millones de años, y no más de doscientos (p. 289).

Si bien los cálculos eran correctos para la física de la época, todo se vino abajo al inicio del siglo XX, cuando se descubrió la radiactividad, lo que condujo a modificar las estimaciones antes aceptadas. El enfriamiento era mucho más lento de lo que supuso Kelvin, debido a los elementos que generan calor por el decaimiento radioactivo. Y la física moderna fue proporcionando, y hasta la fecha, los métodos para determinar la edad de las rocas con base en la radiactividad. En el siglo XX se estableció que la corteza terrestre se consolidó hace más de 4,000 millones de años.

Darwin deja claro sus propias observaciones e interpretaciones geológicas sobre la relación de la erosión con la resistencia de las rocas, la sucesión de estratos, movimientos debidos a fallas geológicas, actividad volcánica, factores que lo llevan siempre a tratar de explicarse el tiempo transcurrido. Lo mismo a partir de la observación de las capas de rocas sedimentarias y su composición, como algunos conglomerados de más de $3000 \mathrm{~m}$ de grosor (p. 271). Y sobre el tiempo geológico:

Quien pueda leer la gran obra de Sir Charles Lyell sobre los principios de la geología, que el historiador futuro reconocerá que ha producido una revolución en la ciencia natural, y después de leerla no admita aún cuán vastos han sido los periodos del tiempo transcurrido, cierre enseguida este volumen (p. 269).

\section{Los fósiles}

Hacia la mitad del siglo XIX la paleontología era una disciplina científica sólida, gracias principalmente a Georges Cuvier (1769-1832), el padre de la misma, quien estudió y clasificó innumerables fósiles y los situó en la secuencia del tiempo. Pero fueron más las controversias por las interpretaciones que se hacían para entender la historia de la Tierra con base en las formas de vida del pasado. Darwin observó los fósiles más con ojos de geólogo que de biólogo, lo que encontramos en varios renglones de El origen de las especies. Los temas no resueltos eran la ausencia de fósiles en muchas formaciones geológicas, o en porciones de ellas, así como las extinciones a lo largo del tiempo. Hace notar la intermitencia entre las formaciones geológicas para explicar que no forzosamente hay una continuidad en el depósito:

Pocos hechos me sorprendieron más, cuando examiné cientos de millas de costas sudamericanas, que se han levantado varios centenares de pies en épocas cercanas, que la ausencia de todo depósito reciente lo bastante extenso para durar por lo menos un breve periodo geológico (p. 275).

Considera que los procesos de transgresión y regresión marina influyen en la conservación o no de los organismos. Las rocas ricas en fósiles deben haberse formado en condiciones de hundimiento, pero con buena provisión de sedimentos que mantengan el mar somero y sepulten y preserven los organismos antes que puedan ser destruidos. Los ambientes de estabilidad en un mar poco profundo, o de levantamiento, no pudieron ser favorables para la conservación de fósiles que, por ejemplo, son escasos en el flysch alpino. Argumenta que las rocas metamórficas e intrusivas están presentes en la superficie terrestre porque fueron despojadas de un manto protector a causa de la erosión (p. 274-277).

Darwin continúa con consideraciones sobre la insuficiencia del registro fósil y las extinciones. Hace ver el error de considerar que la aparición de una especie en una formación 
no significa que no haya existido en otra zona, o que la desaparición de una especie antes de culminar el depósito no significa forzosamente una extinción (p. 278). Para dar peso a sus interpretaciones pone como ejemplo un ambiente geológico moderno:

Cuando las capas como las que fueron depositadas en aguas poco profundas, cerca de la desembocadura del Mississippi, durante alguna parte del periodo glacial, se hayan elevado, los restos orgánicos aparecerán, probablemente por primera vez y desparecerán a niveles diferentes, debido a las migraciones de especies y a los cambios geográficos. Y en el lejano futuro, un geólogo que examinara esos lechos estará tentado a concluir que la duración media de la vida de los fósiles sepultados había sido realmente inferior a la del periodo glacial, en lugar de haber sido realmente mucho mayor, es decir, abarcando desde antes de la época glacial hasta el día actual (p. 279).

\section{Y más adelante menciona:}

Pero este mismo movimiento de descenso tenderá a sumergir la zona de donde se deriva el sedimento, y disminuir así la provisión, mientras el movimiento hacia abajo continúa. A decir verdad, ese equilibrio casi exacto entre la cantidad de sedimentación y el grado de descenso debe ser rara contingencia, pues más de un paleontólogo ha observado que los depósitos muy espesos suelen estar desprovistos de restos orgánicos, salvo cerca de sus límites superiores o inferiores (p. 280).

La disposición de los fósiles en el sentido vertical de los estratos lo explica de manera sencilla:

Cuando la misma especie se presenta en el fondo, en el medio y en lo alto de una formación, lo probable es que no haya vivido en el mismo lugar durante todo el periodo de depósito, sino que haya desaparecido y reaparecido, quizá muchas veces durante el mismo periodo geológico (p. 280).

El análisis de los fósiles convence a Darwin de los cambios de los organismos a través del tiempo. Las formas de vida del Terciario son muy semejantes a las actuales y algunos especialistas concluyeron en este tiempo que no había diferencias, pero otros, con quienes Darwin estaba de acuerdo, afirman que sí las hay, aunque pequeñas, suficientes para dejar claro el proceso evolutivo (p. 282). Considera que

más importante que encontrar un número infinito de esas sutiles formas transicionales que, según nuestra teoría, han vinculado a todas las especies pasadas y presentes del mismo grupo en una larga cadena ramificada de vida (es) buscar solamente unos cuantos eslabones y estos los encontramos con toda seguridad relacionados entre sí (p. 284-285).

El problema de la falta de continuidad en los fósiles llevó a destacados paleontólogos a objetar la transmutación de las especies. Darwin reitera que el registro geológico es imperfecto:

Olvidamos de continuo cuán grande es el mundo comparado con la zona sobre la cual han sido examinadas cuidadosamente las formaciones geológicas; olvidamos que pueden haber existido desde hace tiempo en otras partes grupos de especies, que se hayan multiplicado lentamente antes de invadir los antiguos archipiélagos de Europa y de los Estados Unidos (p. 285).

y señala que el tiempo transcurrido entre uno y otro depósito que dan origen a una formación puede ser superior a la edad de la unidad geológica. De esta manera explica que en un intervalo en que no hay depósito de sedimentos se pudieron multiplicar determinadas especies y al pasar a otra etapa de sedimentación aparecerán los organismos

como creados de pronto (p. 285).

Es el concepto del tiempo en que se apoya Darwin para reforzar sus ideas evolucionistas o es la comprensión del tiempo, resultado de sus observaciones y la lectura de las publicaciones crecientes de su época, lo que lo lleva a la asociación indisoluble de tiempo y evolución.

Pocos años antes los especialistas consideraban que los mamíferos aparecieron en la superficie terrestre de manera súbita al inicio del Terciario. Darwin menciona que en pocos años los fósiles encontrados van modificando esos conceptos. Los mamíferos se reconocen desde el Secundario. Se modificó también la aseveración de Cuvier, en el sentido de que no hay huellas de monos en los estratos terciarios, pero pasaron pocos años para que se identificaran hasta del Mioceno (p. 286).

El tema de los fósiles culmina con un razonamiento que sigue siendo importante en las geociencias: el conocimiento geológico y paleontológico se basaba fundamentalmente en aquella época en estudios en Europa y los Estados Unidos de América:

Paréceme tan imprudente dogmatizar sobre la sucesión de las formas orgánicas en todo el mundo como lo sería un naturalista que después de desembarcar por cinco minutos en algún punto árido de Australia hablara sobre el número y zona que abarcan sus producciones (p. 288).

La interpretación que hace Darwin del pasado a partir del registro de los fósiles, que en aquella época provocaba muchas dudas y escepticismo, es algo que se apega a los conceptos actuales, aunque con modificaciones:

En la naturaleza el proceso será mucho más complicado de lo que está representado en el diagrama, porque los grupos habrán sido más numerosos; habrán durado por periodos extremadamente desiguales de tiempo y se habrán modificado en varios grados. Como sólo poseemos el último volumen del registro geológico y ése muy mutilado, no tenemos derecho a abrigar la esperanza, sino en casos raros, de llenar los amplios intervalos que hay en el sistema natural uniendo así familias u órdenes distintos (p. 307).

\section{Evolución geológica}

Darwin buscó una explicación a un tema fundamental de la geología desde mediados del siglo XIX: el origen y evolución de los continentes y los océanos. Son interesantes las interpretaciones que hace sobre la evolución al considerar que durante el Paleozoico y Mesozoico existieron los mismos océanos que los actuales y los continentes deben haber permanecido donde se encuentran, pero han sufrido cambios sustanciales por oscilaciones desde el Cámbrico, o sea, transgresiones y regresiones marinas. En cambio, supone que antes del Cámbrico pudieron existir continentes donde ahora se extienden los océanos y, a la inversa, 
océanos donde ahora hay continentes (p. 291). La opinión de Darwin es avanzada para su época, cuando se formulan las primeras explicaciones con fundamento científico sobre la evolución de los continentes y océanos, la teoría del geosinclinal, expuesta por J. Hall, precisamente en 1859 , y que creció y evolucionó hasta fines de los años sesenta del siglo $\mathrm{XX}$, cuando fue sustituida por la de la tectónica de placas.

Asimismo, para Darwin la historia geológica no puede explicarse con el detalle que se desearía y lo dice con claridad y sabiduría:

De esta historia poseemos solamente el último volumen, que sólo se refiere a dos o tres países. De este volumen, solamente aquí y allí se ha conservado algún breve capítulo, y de cada página, solamente aquí y allí algunas líneas. Cada palabra del lenguaje que cambia lentamente, y es más o menos diferente en los capítulos sucesivos, puede representar a las formas de vida que se hallan sepultadas (p. 292).

Cuando Darwin escribía El origen de las especies era de amplia aceptación el catastrofismo para explicar los cambios bruscos que se reconocían en la historia de la Tierra a través de los estratos y este es su comentario:

La vieja noción de que todos los habitantes de la Tierra hubieran sido barridos por catástrofes en periodos sucesivos es muy generalmente abandonada, aun por esos geólogos como Elie de Beaumont, Murchison, Barrande, etc., cuyas opiniones generales los conducirían naturalmente a esa conclusión. Al contrario, tenemos todas las razones para creer, por el estudio de las formaciones terciarias, que las especies y grupos de especies desaparecen gradualmente, uno tras otro, primero de un lugar y después de otro, y finalmente del mundo (p. 296).

Una conclusión fundamental de Darwin no tendrá objeciones entre los especialistas, aunque el conocimiento del mundo a que se refiere es naturalmente muy diferente después de 150 años:

He intentado demostrar que el registro geológico es extremadamente imperfecto; que sólo una pequeña porción del globo ha sido explorada geológicamente con cuidado; que sólo ciertas clases de seres orgánicos se han conservado ampliamente en estado fósil; que el número, tanto de especimenes como de especies, conservados en nuestros museos, no es nada comparado con el número de generaciones que deben haber pasado, aun durante una sola formación (p. 315).

A esto agrega la posibilidad de que las especies extinguidas superen con un número muy alto a las conocidas por los movimientos de hundimiento (transgresiones marinas).

\section{La distribución geográfica}

La distribución de los organismos en distintos ambientes tiene relación con su transformación a través del tiempo:

En el caso de esas especies que durante periodos geológicos enteros han sufrido pocas modificaciones, no es muy difícil creer que han migrado desde la misma región, pues durante vastos cambios geográficos y climáticos que han sobrevenido desde antiguos tiempos es casi posible cualquier cantidad de migración (p. 322).

La distribución de organismos semejantes en Eurasia,
África y América llevó a algunos científicos a proponer la migración por puentes $\mathrm{y}$, en el caso de las islas, se consideró que estuvieron unidas con la tierra firme. Darwin aquí no se entusiasma con esta teoría y se refiere a las especies actuales, entre las que son pocas en proporción, que guardan semejanza con las de la era Mesozoica.

Darwin trata de explicar la fauna de continentes y mares considerando, entre otros factores, la posición geográfica. Cuando las islas del mar tienen las condiciones favorables para la migración de organismos de una a otra, la fauna es semejante; cuando se dan condiciones desfavorables, la fauna es totalmente distinta.

La presencia de fauna semejante en las islas del océano se explicaba, por lo general, por relieves preexistentes que las acercaban entre sí o con el continente. Darwin argumenta con abundantes ejemplos que apoyan el concepto de que las especies provienen de una fuente común:

Entonces todos los grandes hechos principales de la distribución geográfica son explicables con la teoría de la migración, junto con la posterior modificación y multiplicación de las nuevas formas. Podemos entender así la alta importancia de las barreras, sean de agua o de tierra, no sólo para separar, sino aparentemente para formar también las diversas provincias zoológicas y botánicas (p. 361).

La relación entre las islas no es sólo la distancia entre ellas, sino la profundidad del mar, las corrientes del mismo, los huracanes. Las migraciones son un proceso continuo a lo largo de la historia de la Tierra y se relacionan de manera estrecha con la evolución de los organismos y Darwin lo explica con apoyo en la geología:

Y cuando conozcamos mejor los muchos medios de migración, entonces, con la luz que nos da y seguirá dándonos la geología sobre los antiguos cambios de clima y de nivel de la Tierra, podremos seguramente reconstruir de un modo admirable las antiguas migraciones de los habitantes del mundo (p. 427).

Supone que muchas islas hundidas en el mar pudieron servir como puentes y lo fundamenta en una de las contribuciones más importantes que hizo a la geología: el origen de los anillos de coral (atolones) a causa del hundimiento de las islas (ascenso del nivel del mar) y deja en claro el tema, lo que sigue siendo válido en nuestra época:

Y la composición casi universalmente volcánica de tales islas, tampoco favorece la admisión de que sean restos de continentes hundidos; si hubieran existido originalmente como cadenas de montañas continentales, algunas por lo menos de las islas habrían estado formadas, como las otras cimas de montañas, de granito, esquistos metamórficos, viejas rocas fosilíferas y otras en lugar de consistir en meras masas de materia volcánica (327).

Con el tiempo, un siglo después, quedaría claro que la constitución de las islas volcánicas no era algo local y casual, sino expresión de la mayor parte del piso profundo del océano mundial.

Menciona las glaciaciones como algo que motiva las migraciones. Al bajar la temperatura en una región la fauna buscará en otro lugar las condiciones semejantes, principalmente hacia el sur. Cuando encuentren barreras naturales pueden sufrir extinción. 
Explica que las formas de vida en alguna época se desplazaron desde el norte y otra desde el sur, en dirección al ecuador, pero con mayor influencia la que se produce de norte a sur. Y continúa con una nota, donde hace una analogía entre biología y geología:

Así como la marea deja su marca en líneas horizontales, que suben más en las costas donde más altas son las mareas, así el océano de la vida ha dejado su resaca viva en la cumbre de nuestras montañas, en una línea que sube suavemente desde las tierras bajas árticas hasta una gran altura, bajo el ecuador (p. 344).

Es bien conocido que las observaciones de Darwin en las islas Galápagos fueron un sustento poderoso a la teoría de la evolución. Allí encontró más argumentos para hacer inconsistente la teoría entonces aceptada, de la aparición de las especies de manera independiente. Las islas tuvieron que ser pobladas por migraciones, aunque fue un proceso difícil por las condiciones geográficas y geológicas. Al mismo tiempo hubo un aislamiento que dio lugar a la transformación de los organismos, diferenciando especies de una isla a otra. Puso en claro, tres temas relacionados: origen de las islas, poblamiento y aislamiento (p. 358).

\section{A 150 años}

Darwin continuó el estudio de la naturaleza que ya era rico a fines del siglo XVIII. Entre otros hechos, fue de especial importancia que las publicaciones geológicas, principalmente de Lyell, le proporcionaran los fundamentos para su formación como geólogo y comprender la Tierra a partir de un proceso evolutivo.

El creacionismo fue en el pasado la explicación del Universo, incluyendo nuestro planeta, su relieve y la vida que sostiene. Los estudios de la naturaleza, principalmente desde el siglo XVIII, fueron cambiando los conceptos tradicionales. Entre los sucesos más importantes está la teoría sobre el origen del Sistema Solar, expuesta por separado por Immanuel Kant (1724-1804) y Pierre Simon marqués de Laplace (1749-1827). Lyell dejó en claro que nuestro planeta es resultado de un proceso evolutivo prolongado. En la medida en que se fue acumulando información relacionada con la Tierra y la vida, llegó el momento en que por separado Darwin y Wallace elaboraron la teoría de la selección natural. Fue la culminación de una de las etapas más importantes de lectura de la naturaleza. A partir de 1859 la teoría de la evolución, cuyo padre es J.B. Lamarck, se reforzó y pasó a una etapa de crecimiento irreversible que continúa en la primera década del siglo XXI.

Hoy día hay controversias sobre el mecanismo con el que se producen cambios en los organismos, pero no hay duda alguna sobre la evolución, la han fortalecido los avances de la biología y la geología posterior a Darwin. Es simplemente la única teoría científica al respecto. El creacionismo resultó en el pasado de la incomprensión del hombre del tiempo de la Tierra, de concebir el mundo en función del tiempo humano, del tiempo de la memoria. histórica que no llega a una decena de miles de años.
La influencia de Darwin en el medio académico fue tal que surgió el término darwinismo. Los conceptos evolucionistas y la lucha por la vida fueron aplicados en otras disciplinas. Por ejemplo, la teoría de William M. Davis El ciclo geográfico, publicada en 1899, que para muchos autores representa el surgimiento de la ciencia del relieve terrestre, la Geomorfología, está inspirada en Darwin. Se trata de un ciclo evolutivo idealizado del relieve y que sólo parcialmente corresponde con la realidad. El darwinismo actual puede reflejar o no el pensamiento del científico británico, él es ajeno a las interpretaciones que se han hecho de su obra.

\section{Reconocimientos}

El autor agradece los comentarios de dos árbitros anónimos. Este trabajo es parte del proyecto de investigación Geografía e historia natural: hacia una historia comparada. Los estudios mexicanos, con apoyo del PAPIIT y a cargo de la doctora Luz Fernanda Azuela Bernal. Ailsa Winton y Lorenzo Vázquez revisaron una parte de la redacción.

\section{Bibliografía}

Darwin C., 2004, El origen de las especies: El origen de las especies: México, D.F., México, Porrúa, 455 p.

Farrington, B., 1979, El evolucionismo: Barcelona, España, LAIA, $121 \mathrm{p}$.

Gribbin, J., 2003, Historia de la Ciencia 1543-2001: Barcelona, España, Crítica, $552 \mathrm{p}$.

Herbert, S., 2005, Charles Darwin, Geologist: New York, U.S.A., Cornell University, $485 \mathrm{p}$.

Romer, A.S., 1985, Darwin y el registro fósil, en Barnett, S.A. (ed.), Un siglo después de Darwin. 2. El origen del hombre: Madrid, Alianza Editorial, 61-88.

Ruiz, R., Ayala, F.J, 1998, El método en las ciencias. Epistemología y darwinismo: México, D.F., México, Fondo de cultura Económica, $216 \mathrm{p}$.

Ruiz, R., Ayala, F.J., 2002, De Darwin al DNA y el origen de la humanidad: la evolución y sus polémicas: México, D. F., México, Fondo de cultura Económica/UNAM, 293 p.

Sarukhán, J, 1988, Las musas de Darwin: México, D.F., México, Fondo de Cultura Económica, $261 \mathrm{p}$.

Torres, J.L., 1995, En el nombre de Darwin: México, D. F., Fondo de Cultura Económica, $159 \mathrm{p}$.

Yonge, C.M., 1985, Darwin y los arrecifes de coral, en Barnett S.A. (ed.), Un siglo después de Darwin. 2. El origen del hombre: Madrid, España, Alianza Editorial, 157-183.

Manuscrito recibido: 5/2/2009

Manuscrito corregido: 7/10/2009

Manuscrito aceptado: 14/10/2009 